\title{
Natural history of hepatic hemangiomas as a guide for surgical indication
}

\author{
Mohamed M. Mogahed ${ }^{1}$, Ashraf Anas Zytoon ${ }^{2^{*}}$ (D, Bassem Essa ${ }^{3}$, Wessam Abdellatif ${ }^{4}$, Nashwa Ghanem ${ }^{5}$ and \\ Bahaa ElWakeel ${ }^{1}$
}

\begin{abstract}
Background: Generally, hemangiomas are asymptomatic and most lesions are incidentally diagnosed on abdominal ultrasonography. The indications for surgery are still debated mainly due to limited data about the natural history of hepatic hemangiomas. The aim of this study is to investigate the natural history of hepatic hemangiomas, the effects of age and sex on their growth, and their tendency to increase in size or causing symptoms or complications as a guide for surgical indication. In this current study, combined retrospective and prospective analysis was conducted on 186 Egyptian adult patients having 244 hepatic hemangiomas who attended to the outpatient clinics. Follow-up was performed to observe the accurate site, size, number, rate of growth of the lesions, and their tendency to cause symptoms or complications.

Results: Ninety-one percent of patients were asymptomatic and $9 \%$ were symptomatic. During follow-up, $48 \%$ of patients showed an increase in tumor diameter, 22\% decreased, and 30\% were stable. The growth rate at 18-45-year group showed an increasing trend that was higher in females; it was $3.3 \pm 2 \mathrm{~mm} /$ year for males and $3.9 \pm 1.8 \mathrm{~mm} /$ year for females. At > 45 year group, the female patients showed a decreasing trend $(-2.1 \pm 1.1 \mathrm{~mm} /$ year) while in males showed an increasing trend that was slower than in 18-45-year group (2.6 $\pm 1.7 \mathrm{~mm} /$ year).

Conclusion: Growth pattern of liver hemangiomas is affected by age and sex. The majority of hemangiomas are asymptomatic and complications are rare. Hemangioma size alone is not an indication for surgery in asymptomatic patients. Surgical indications are limited to patients with severe symptoms, complications, or suspicious lesions. Most hemangiomas can be managed conservatively even giant hemangiomas.
\end{abstract}

Keywords: Hepatic hemangiomas, Natural history, Surgical indication

\section{Background}

Hepatic hemangioma is the most common benign tumor of the liver [1], with an incidence reach up to $7.3 \%$ on autopsy [2] and up to $20 \%$ in the general population [3]. This tumor is typically observed in females with female to male ratio that may reach 5:1 at age of 50 years [4]. Hepatic hemangiomas are usually asymptomatic and diagnosed incidentally on abdominal ultrasonography [5]. MRI has high sensitivity and specificity for diagnosis of small and atypical hepatic hemangiomas [5]. The indications for

\footnotetext{
*Correspondence: ashradio@gmail.com

${ }^{2}$ Radiodiagnosis Department, Faculty of Medicine, Menoufia University, Menoufia, Egypt

Full list of author information is available at the end of the article
}

surgery are still not clearly defined up till now. The main reason is the limited and inconsistent data available on the natural history of hepatic hemangiomas and on their tendency to increase in size or causing complications [6]. Ultrasonography is the first diagnostic and most available imaging test. US shows the hemangioma as well-defined, occasionally lobulated, homogeneously hyperechoic with posterior acoustic enhancement, and frequently located close to a hepatic or a portal vein with sensitivity of $96.9 \%$ and specificity of $60.3 \%$ [7]. Color-Doppler US does not improve accuracy in diagnosis, and it only shows blood flow in liver hemangioma with an intralesional arterioportal shunt $[8,9]$. Liver hemangiomas appears by CT as well-demarcated hypo-dense masses that is showing

\section{Springer Open}

(0) The Author(s). 2020 Open Access This article is licensed under a Creative Commons Attribution 4.0 International License, which permits use, sharing, adaptation, distribution and reproduction in any medium or format, as long as you give appropriate credit to the original author(s) and the source, provide a link to the Creative Commons licence, and indicate if changes were made. The images or other third party material in this article are included in the article's Creative Commons licence, unless indicated otherwise in a credit line to the material. If material is not included in the article's Creative Commons licence and your intended use is not permitted by statutory regulation or exceeds the permitted use, you will need to obtain permission directly from the copyright holder. To view a copy of this licence, visit http://creativecommons.org/licenses/by/4.0/. 
arterial peripheral nodular enhancement followed by progressive centripetal homogeneous filling toward the center at delayed images in triphasic post contrast study; however, small lesions of liver hemangiomas appears with cystic areas, fibrosis, or thrombosis may show atypical patterns [10]. CT has a sensitivity of $98.3 \%$ and a specificity of 55\% [7]. Dynamic MRI is the best diagnostic imaging for liver hemangioma with sensitivity of $100 \%$ and specificity of $85.7 \%$ [7]. MRI shows a smooth, welldefined, homogenous lesion, hypointense on T1 and hyperintense on T2-weighted images. Some malignant hepatic lesions may show a similar hyperintensity on T2; to differentiate liver hemangioma from solid neoplastic liver lesions, the echo time is increased which causes signal from malignant lesions to decrease and signal from liver hemangioma to increase. With gadolinium administration, hemangioma shows a peripheral enhancement on arterial phase and contrast retention on delayed phases; this allows differentiation from hypervascular tumors that have a contrast washout on delayed phase [11].

This study aimed to investigate the natural history of hepatic hemangiomas and the effects of age and sex on their growth as a guide for surgical indications.

\section{Methods}

\section{Study population}

This combined retrospective and prospective study from April 2014 to March 2020 on 186 adult patients (54 males and 132 females) with 244 hepatic hemangioma lesions. The median age of patients was 45 years and the range was $18-68$ years.

\section{Patient inclusion criteria}

Adult patients (age is more than 18 years) having hepatic hemangioma.

\section{Patient exclusion criteria}

Patients younger than 18 years, females receiving oral contraceptive pills, and patients who had received treatment for the hemangiomas were excluded.

Follow-up was performed to observe the accurate site, size, number, rate of growth of the lesions, and their tendency to cause symptoms or complications. Diagnosis of hemangiomas was done by typical ultrasonographic findings and patients with atypical manifestations underwent enhanced computed tomography or magnetic resonance imaging.

Patients were divided into two age groups: $18-45$ years and $>45$ years. Data regarding sex, age, tumor diameter, and follow-up period were recorded. The maximum diameter was used to assess the tumor size. The change of tumor growth was expressed in $\mathrm{mm} /$ year. Ultrasound examinations for all patients were done every 6 months for at least 2 times after the initial diagnosis.
Follow-up period of 12 to 24 months was performed in the outpatient clinics, with repeated US and blood examinations (complete blood count, prothrombin time and liver function tests) every 6 months. Giant hemangiomas were defined in the current study as hemangiomas with a diameter $>10 \mathrm{~cm}$ [7].

Written consent form was filled by every patient after detailed explanation of the study and the plane of management.

The study was approved by the Ethics Review Committee of our institute.

\section{Study design}

Combined retrospective and prospective study was conducted on 186 adult patients (more than 18 years) having 244 hepatic hemangiomas who attended to the outpatient clinics, 54 males and 132 females. Follow-up was performed to observe the accurate site, size, number, rate of growth of the lesions, and their tendency to cause symptoms or complications.

\section{Imaging proctocol}

Ultrasound examination was performed by ultrasound scanner (EPIQ 7 Machine-Philips ultrasound). Ultrasonography of the whole liver parenchyma was done with several imaging access, and many application techniques were used during examination (subcostal diagonal, subcostal longitudinal, sagittal and transverse, right intercostal lateral plans). The selection of planes depends on the accessibility of the lesion by the ultrasound beam intraabdominally away from ribs and gas containing viscera.

CT examination was performed by CT scanner (Aquilion PRIM-Canon Medical). Triphasic CT scanning of the liver where performed at $120 \mathrm{kvp}$ and 200-250 mAs. Patients were fasting for $5 \mathrm{~h}$ before procedure then were given non-ionic I/ $\mathrm{V}$ contrast of $1.5 \mathrm{ml} / \mathrm{kg}$ with dose ranging from 80 to $100 \mathrm{ml}$. Patient preparation also included administration of $1000 \mathrm{ml}$ of water 30-60 min prior to the examination as oral contrast. First, we scan all the liver before I/V contrast injection (non-contrast images) then after injection of intravenous contrast material, liver was scanned in the arterial phase for 15-35 $\mathrm{s}$, the portal was scanned for $60-70 \mathrm{~s}$, venous phase for 70-90 s, and delayed scanning after 5-10 min).

MRI examination was performed by MRI scanner (Ingenia Philips Medical Systems). Technique of MRI requires numerous sequences and imaging at multiple times after the administration of contrast at different axial and coronal plans. T2-weighted sequence: T2 HASTE and T2 fat-saturated, non-contrast T1-weighted sequences: T1 inphase and out-of-phase, T1 fat-saturated, diffusionweighted imaging (DWI): fat-saturated single-shot diffusion-weighted EPI, post-contrast dynamic sequences T1 2D or 3D gradient-echo sequences: arterial phase: 20 
$30 \mathrm{~s}$, portal venous phase: $60-70 \mathrm{~s}$, equilibrium phase: $5-$ $10 \mathrm{~min}$, with and without fat sat.

We used the longitudinal, sagittal, and transverse plans for measuring the size of lesions by ultrasonography. By $\mathrm{CT}$ and MRI, we estimated the volume of the lesion from the axial and coronal images by measuring the antero-posterior, transverse, and longitudinal diameters.

\section{Statistical analysis}

Statistical analysis was performed using SPSS, Version 23 (SPSS Inc., Chicago, IL). Chi-square test was used to compare between the groups with respect to categorical data. Comparisons between two groups for normally distributed numeric variables were done using Student's $t$ test while for non-normally distributed numeric variables, comparisons were done by Mann-Whitney $U$ test. Numerical variables were presented as mean \pm SD. Descriptive statistics were reported as frequencies and percent.

\section{Results}

Of the 186 patients (54 males and 132 females; male/female ratio was 1:2.4) having 244 lesions, 137 had single lesion and 49 had more than 1 lesion. The smallest hemangioma was $1 \mathrm{~cm}$, and the largest was $19 \mathrm{~cm}$ in diameter with median $4.7 \mathrm{~cm}$.

During the median 16 (range 12-30) months followup, 89 patients (48\%) showed an increase in tumor diameter, 41 (22\%) tumor diameters decreased, and 56 (30\%) tumor diameters were stable. Patient data are summarized in Table 1

The current study showed that hemangioma more frequently affects the right lobe than the left lobe as 178 $(73 \%)$ of hemangiomas were in the right lobe and 66 (27\%) were in the left lobe.

Typical appearance of hemangioma by ultrasound was detected in 151 patient that all of them presented by a well-defined, lobulated and homogeneously hyperechoic lesions that is showing posterior acoustic enhancement, some of them located close to a hepatic or a portal vein. 35 patients presented by atypical findings, some lesions showed homogenous hypoechoic texture and other lesions showed peripheral high echogenicity and hypoechoic center, according to these atypical findings we decided to do further imaging modalities to confirm diagnosis (Fig. 1).

Twenty patient did triphasic CT and lesions gives typical features that is seen hypo-attenuating to hepatic parenchyma at non contrast images, then gives discontinuous, nodular, peripheral enhancement at the arterial phase followed by progressive peripheral enhancement with centripetal fill-in at portal and venous phases, finally at delayed phase lesions showed irregular fill-in and therefore become iso- or hyper-attenuating to
Table 1 Data of hepatic hemangioma patients included in the study

\begin{tabular}{|c|c|}
\hline Total number of patients & 186 \\
\hline Males & 54 \\
\hline Females & 132 \\
\hline Age (median, range) years & 45, (18-68) \\
\hline Total number of hemangioma lesions & 244 \\
\hline Number of patients with single lesion & 137 \\
\hline Number of patients with multiple lesions & 49 \\
\hline Hemangioma diameter (median, range) cm & $4.7(1-21)$ \\
\hline \multicolumn{2}{|l|}{ Hemangioma location (total = 244 lesion) } \\
\hline Right lobe & $178(73 \%)$ \\
\hline Left lobe & $66(27 \%)$ \\
\hline Surface hemangioma & $212(87 \%)$ \\
\hline Intraparenchymatous hemangioma & $32(13 \%)$ \\
\hline \multicolumn{2}{|l|}{ Hemangioma growth pattern (number, \%) } \\
\hline Increased in diameter & $89(48 \%)$ \\
\hline Decreased & $41(22 \%)$ \\
\hline Stable & $56(30 \%)$ \\
\hline \multicolumn{2}{|l|}{ Clinical presentation (number, \%) } \\
\hline Asymptomatic & $169(91 \%)$ \\
\hline Symptomatic & $17(9 \%)$ \\
\hline Patients with hemangioma diameter $\geq 10 \mathrm{~cm}$ & $35(19 \%)$ \\
\hline \multicolumn{2}{|c|}{ Growth rate at (18-45-year group) mm/year, (mean \pm SD) } \\
\hline Males & $3.3 \pm 2$ \\
\hline Females & $3.9 \pm 1.8$ \\
\hline \multicolumn{2}{|l|}{ Growth rate at (> 45-year group) mm/year } \\
\hline Males & $2.6 \pm 1.7$ \\
\hline Females & $-2.1 \pm 1.1$ \\
\hline
\end{tabular}

Data are presented as number and \% unless otherwise indicated

hepatic parenchyma (Fig. 2). Fifteen patients did dynamic MRI and all lesions gives the typical features of hemangioma by MRI, all lesions was relatively hypointense to liver parenchyma at T2 images, hyperintense to liver parenchyma at T2 images, at dynamic sequences all lesions showed peripheral nodular discontinuous enhancement that is progresses centripetally toward the center at portal images, then retained contrast on delayed images, all was hyperintense on diffusionweighted imaging due to slow blood flow, and hyperintense or mixed (hyper and hypointense regions) on ADC map (Fig. 3).

The majority of patients with liver hemangiomas were asymptomatic, 169 patients (91\%), and most of the lesions were diagnosed during physical examination and investigations for other gastrointestinal diseases and abdominal discomfort. Seventeen patient were symptomatic (9\%), 6 of those 17 patients had severe symptoms in the form of abdominal pain, distension and discomfort 
while 11 of them had mild symptoms. The six patients with severe symptoms were fully investigated to exclude other gastrointestinal causes of their symptoms. 19\% of patients $(35 / 186)$ had hemangioma $10 \mathrm{~cm}$ diameter or more, all symptomatic patients were in this group $(17 / 35=48.5 \%)$, while $(18 / 35=51.5 \%)$ were asymptomatic in spite of the large diameter of hemangioma (Fig. $4)$.

Nineteen percent of patients (35/186) had hemangioma $10 \mathrm{~cm}$ diameter or more; all symptomatic patients were in this group $(17 / 35=48.5 \%)$, while $(18 / 35=51.5 \%)$ were asymptomatic in spite of the large diameter of hemangioma.

At the time of diagnosis, serum laboratory tests were within normal levels in 90.3\% (168/186) of patients. Eighteen patients had slight elevation in liver transaminases, alkaline phosphatase, or bilirubin.

\section{Growth pattern of hepatic hemangiomas}

The median age of patients was 45 years and the range was $18-68$ years. Patients were subdivided according to their age into two groups (18-45 and more than 45 years) to describe the effect of age and sex on hemangioma growth pattern.

The growth rate at 18-45-year group showed an increasing trend that was higher in females than in males. It was $3.3 \pm 2 \mathrm{~mm} /$ year for males and $3.9 \pm 1.8 \mathrm{~mm} /$ year for females in this group.

The growth rate at (> 45-year group) the female patients showed a decreasing trend $(-2.1 \pm 1.1 \mathrm{~mm} /$ year $)$ with regression of hemangioma size most obvious between the ages of 55-68 years while in males, it showed an increasing trend that was slower than in 18-45-year group with $2.6 \pm 1.7 \mathrm{~mm} /$ year.

As regards the 17 symptomatic patients, 11 patients with mild symptoms continued follow-up and responded to medical treatment of other associated gastrointestinal disorders and reassurance. Six patients with severe symptoms, 2 patients refused the operation and continued follow-up, 4 patients underwent surgery, 2 underwent enucleation, and 2 underwent liver resection with no postoperative complications or mortality. One asymptomatic case was suspicious and malignancy could not be excluded by imaging; the patient underwent surgery and proved to be negative for malignancy. Data of patients underwent surgery are summarized in Table 2.

Surgically treated lesions were huge, occupying nearly most of the right or left lobe and not only segment and were mainly peripheral.

No spontaneous or traumatic intraperitoneal hemorrhage occurred in the current study, and no recorded KasabachMerritt syndrome as it is a rare condition recorded only in children and our cases were adults.

\section{Discussion}

In the current study during follow-up, $48 \%$ of patients showed an increase in tumor diameters, $22 \%$ tumor diameters decreased, and 30\% tumor diameters were stable. This is comparable to results of Ankang Wang et al.'s study who reported $40.3 \%$ of patients showed increased diameter, $40.6 \%$ decreased, and $19.1 \%$ stable. While in Jing L et al.'s study, $61.0 \%$ patients had hemangiomas that increased in size; $23.7 \%$ had stable lesions, and $8.5 \%$ decreased in size $[12,13]$.

Studies have reported that hepatic hemangioma is more likely to occur in female patients, and long exposure to estrogen promotes the growth of hepatic hemangioma [14]. In this study, male:female ratio was 1: 2.4. Age is an important factor affecting the growth rate of hepatic hemangiomas [15]. In the current study, the growth before the age of 45 years showed an increasing trend that was higher in females than in males and after the age of 45 years the female patients showed a decreasing trend with regression of hemangioma size most obvious between the ages of 55 and 68 years while in males showed an increasing trend that was slower than in 18-45-year group.

The role of female gender in hemangioma growth has been discussed in a long-term follow-up study of Ankang Wang which revealed that there were significant differences in hemangioma growth between males and females [12].
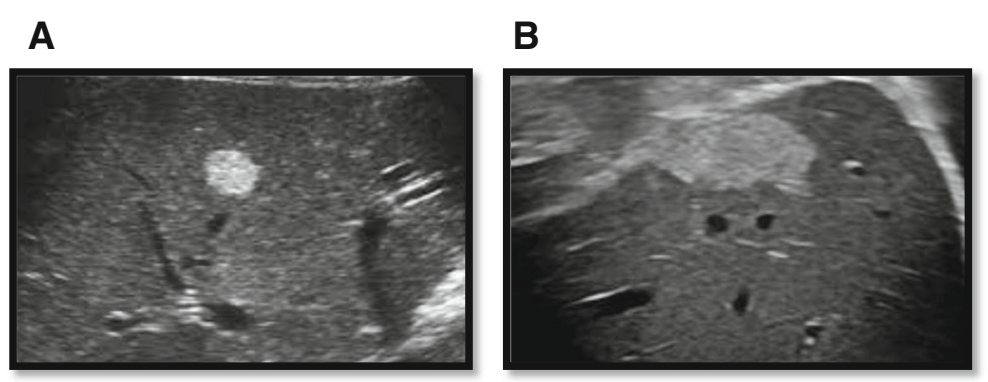

Fig. 1 Hemangioma as seen by ultrasonography. a Right lobe segment $V$ well-defined hyper-echoic lesion close to hepatic vein. $\mathbf{b}$ Left lobe subcapsular segment II hyperechoic lesion 


\section{A}

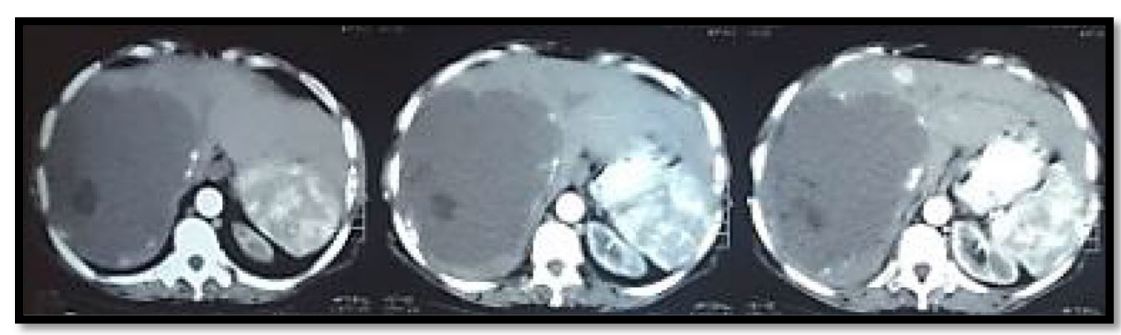

B

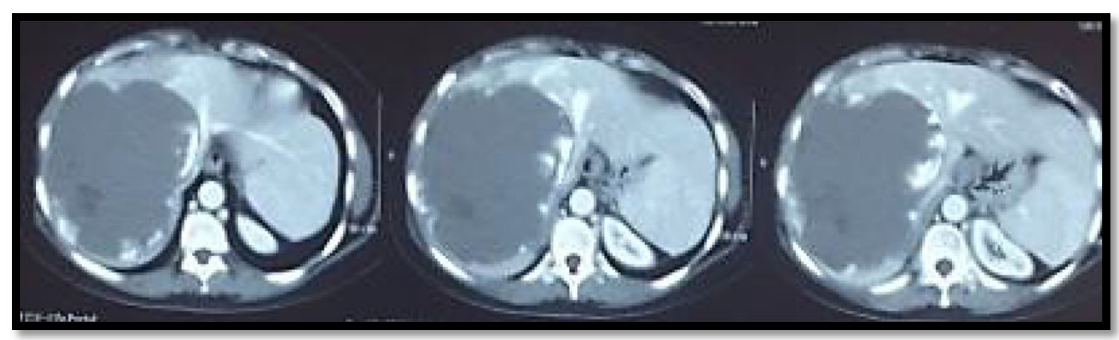

C

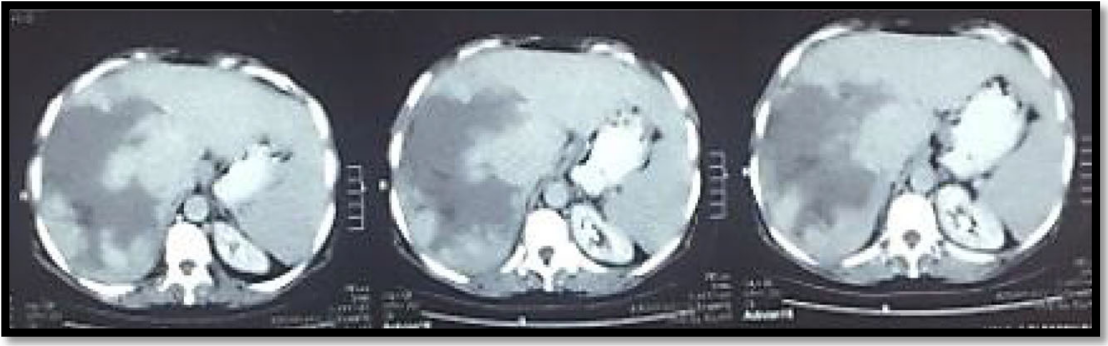

Fig. $\mathbf{2}$ Large right hepatic lobe hemangioma as seen by triphasic CT. a Arterial phase showing faint peripheral enhancement of the lesion. $\mathbf{b}$ Porto-venous phase showing more pronounced peripheral nodular enhancement by the lesion. c Delayed images showing progressive contrast enhancement toward the center of the lesion

Abdominal pain and discomfort caused by distension of the liver capsule are the most common presentations of symptomatic hepatic hemangioma. In the current study, $91 \%$ of patients with liver hemangiomas were asymptomatic and $9 \%$ were symptomatic with no mortality or complications during the follow-up period. This is comparable to results of Jing $L$ et al.'s study who reported that 21 of 220 patients (9.5\%) were symptomatic while in Ankang Wang's study, the symptomatic patient were about $6 \%[12,13]$.

Some authors define the hemangioma as giant if more than $5 \mathrm{~cm}$, and others define giant hemangiomas if more than $10 \mathrm{~cm}[16,17]$. Nineteen percent of the current study patients $(35 / 186)$ had hemangioma diameter of > $10 \mathrm{~cm} ; 51.5 \%$ were asymptomatic in spite of the large diameter of hemangioma and need no treatment but only observation, while $48.5 \%$ were symptomatic. This goes with Boukerrouche A's study who concluded that size of the tumor alone is not indication for surgery [18].

Exclusion of other causes of abdominal pain in patient with liver hemangioma is mandatory. As in many recorded studies like in Farges and colleagues' study, pain disappeared in $54 \%$ of patients after treatment of

Table 2 Data of the five patients who underwent surgery for hepatic hemangioma

\begin{tabular}{lllllllll}
\hline $\boldsymbol{n}$ & Age (years) & Gender & Tumor $(\mathbf{c m})$ & Surgical indication & Surgical approach & Site & Relief of symptom & Complications \\
\hline 1 & 57 & Female & 12.7 & Abdominal pain & Enucleation & Right lobe & Yes & No \\
2 & 53 & Male & 11.3 & Abdominal pain & Enucleation & Right lobe & Yes & No \\
3 & 44 & Female & 13.1 & Abdominal discomfort & Laparoscopic resection & Left lobe & Yes & No \\
4 & 49 & Female & 19 & Abdominal pain & Open resection & Left lobe & Yes & No \\
5 & 58 & Male & 10.5 & Atypical lesion & Open resection & Right lobe & Yes & No \\
\hline
\end{tabular}



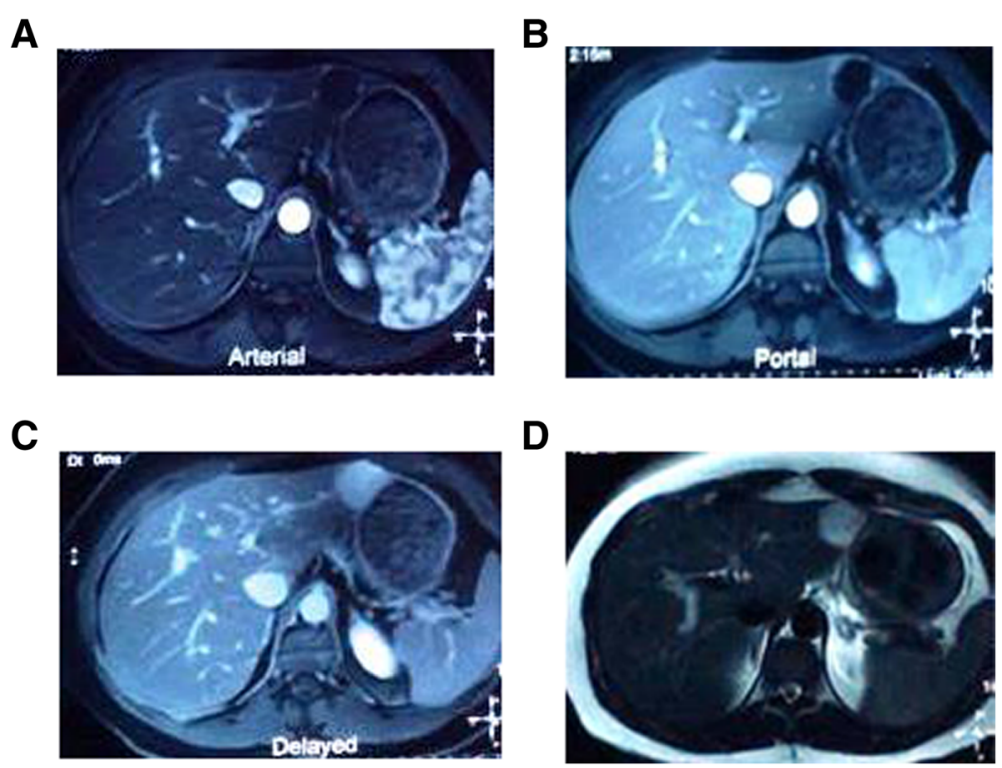

Fig. 3 Axial dynamic MRI sequences of the liver for left lobe $3 \mathrm{~cm}$ sub-capsular hemangioma. a Axial T1 arterial phase showing faint peripheral enhancement. b Progressive enhancement at axial T1 portal phase. c Axial T1 delayed phase showing homogenous enhancement of the lesion. d The lesion showing bright signal at axial T2 images

associated disorders and in 4/11 patients, pain persisted even after tumor resection [19].

Many patients after incidental diagnosis of liver hemangioma get anxiety. Anxiety alone should never be considered as indication for surgery [20]. Malignant transformation is practically inexistent and the patients have to be reassured. Patients operated for hemangioma because of anxiety continued to have anxiety after surgery $[21]$.
Spontaneous hemorrhage and Kasabach-Merritt syndrome are rare [22, 23]. In the current study, there was no spontaneous or traumatic intraperitoneal hemorrhage occurred, no recorded Kasabach-Merritt syndrome as it is recorded in children and our cases were adult. This is comparable to results of Jing L et al.'s study with no reported cases, while Akang Wang's study reported only one case of 590 hepatic hemangioma patients diagnosed as spontaneous internal hemorrhage at surgery $[12,13]$.

\section{Frequency of asymptomatic and symptomatic patients}

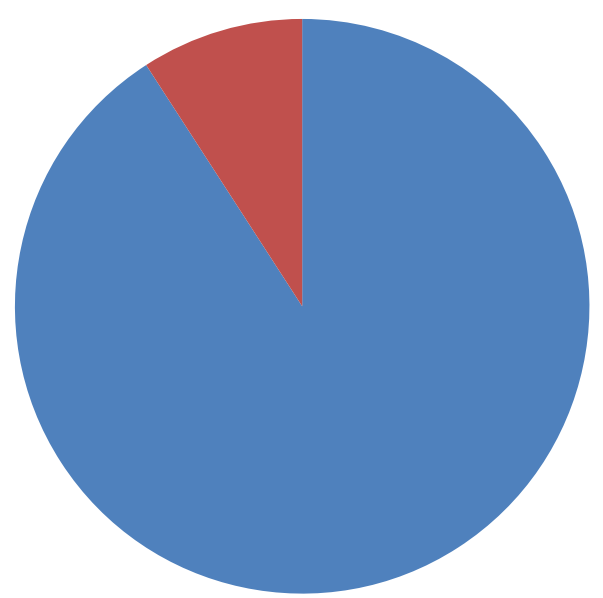

Asymptomatic patients 169 (91\%)

- Symptomatic patients 17 (9\%)

Fig. 4. Frequency of asymptomatic and symptomatic hemangioma patients 


\section{Conclusion}

Growth pattern of hemangiomas is affected by age and sex. The majority of hepatic hemangiomas are asymptomatic and complications are rare. Hemangioma size alone is not indication for surgery in asymptomatic patients. Surgical indications are limited to patients with severe symptoms, complications, or suspicious lesion. Most hemangiomas, even giant hemangiomas, can be managed conservatively.

\section{Abbreviations}

$\mathrm{CT}$ : Computed tomography; MRI: Magnetic resonance imaging

\section{Acknowledgements}

N/A

\section{Authors' contributions}

M. M. M., A. A. Z., B. E., W. AL., N. G., and B. EW. made substantial contribution to the conception of the study; substantial contribution to the design of the study; substantial contribution to the acquisition and analysis of the data; substantial contribution to the interpretation of data; substantial contribution to the creation of the final work; substantial contribution to the study revision; and substantial contribution to the accuracy or integrity of the submitted manuscript. All authors have read and approved the manuscript.

\section{Funding}

None

Availability of data and materials

The data supporting the results is available.

\section{Ethics approval and consent to participate}

The study was approved by the Research Ethics Committee of our medical institute (committee's reference number: ITH00121; date: 19 February 2020). All study procedures were carried out in accordance with the Declaration of Helsinki regarding research involving human subjects. Written consent form was filled by every patient after detailed explanation of the study and the plane of management

\section{Consent for publication}

N/A

\section{Competing interests}

The authors declare that they have no competing interests.

\section{Author details}

${ }^{1}$ Departments of Surgery, NHTMRI, Cairo, Egypt. ${ }^{2}$ Radiodiagnosis Department, Faculty of Medicine, Menoufia University, Menoufia, Egypt. ${ }^{3}$ Tropical Medicine Department, NHTMRI, Cairo, Egypt. ${ }^{4}$ Radiology Department, NHTMRI, Cairo, Egypt. ${ }^{5}$ Internal Medicine Department, Faculty of Medicine, Cairo University, Cairo, Egypt.

Received: 31 August 2020 Accepted: 13 November 2020

Published online: 30 November 2020

\section{References}

1. Yin X, Huang X, Li Q et al (2018) Hepatic hemangiomas alter morphometry and impair hemodynamics of the abdominal aorta and primary branches from computer simulations. Front Physiol 9:334

2. Herman P, Costa ML, Machado MA et al (2005) Management of hepatic hemangiomas: a 14-year experience. J Gastrointest Surg 9:853-859

3. Bahirwani R, Reddy KR (2008) The evaluation of solitary liver masses. Aliment Pharmacol Ther. 28:953-965

4. Duxbury MS, Garden OJ (2010) Giant haemangioma of the liver: observation or resection? DigSurg 27:7-11

5. Caseiro-Alves F, Brito J, Araujo AE et al (2007) Liver haemangioma: common and uncommon findings and how to improve the differential diagnosis. Eur Radiol 17:1544-1554
6. Giuliante F, Ardito F, Vellone M et al (2011) Reappraisal of surgical indications and approach for liver hemangioma: single center experience on 74 patients. Am J Surg 201:741-748

7. Toro A, Mahfouz AE, Ardiri A et al (2014) What is changing in indications and treatment of hepatic hemangiomas. A review. Ann Hepatol. 13:327-339

8. Perkins AB, Imam K, Smith WJ, Cronan JJ (2000) Color and power Doppler sonography of liver hemangiomas: a dream unfulfilled ? J Clin Ultrasound. 28:159-165

9. Lim KJ, Kim KW, Jeong WK et al (2012) Colour Doppler sonography of hepatic haemangiomas with arterioportal shunts. Br J Radiol 85:142-146

10. Yamashita Y, Ogata I, Urata J, Takahashi M (1997) Cavernous hemangioma of the liver: pathologic correlation with dynamic CT findings. Radiology 203: $121-125$

11. McFarland EG, Mayo-Smith WW, Saini S, Hahn PF, Goldberg MA, Lee MJ (1994) Hepatic hemangiomas and malignant tumors: improved differentiation with heavily T2-weighted conventional spin-echo MR imaging. Radiology 193:43-47

12. Wang A, Deng J, Qian B et al (2019) Natural history of hepatic hemangioma: a follow-up analysis of 534 patients. Frontiers in Life Science 12:27-32

13. Jing $L$, Liang $H$, Caifeng $L$ et al (2016) New recognition of the natural history and growth pattern of hepatic hemangioma in adults. Hepatol Res 46:727-733

14. Van Malenstein H, Maleux G, Monbaliu D et al (2011) Giant liver hemangioma: the role of female sex hormones and treatment. Eur J Gastroenterol Hepatol 23:438-443

15. Liu X, Yang Z, Tan H, Xu L, Liu L, Huang J, Si S, Sun Y, Zhou W (2018) Patient age affects the growth of liver haemangioma. HPB 20:64-68

16. Grieco MB, Miscall BG (1978) Giant hemangiomas of the liver. Surg Gynecol Obstet 147:783-787

17. Van Tilborg AA, Nielsen K, Scheffer HJ et al (2013) Bipolar radiofrequency ablation for symptomatic giant $(>10 \mathrm{~cm}$ ) hepatic cavernous haemangiomas: initial clinical experience. Clin Radiol 68:e9-e14

18. Boukerrouche A (2017) Management of giant liver hemangioma. J Gastroenterol Dig Dis. 2:12-16

19. Farges O, Daradkeh S, Bismuth H (1995) Cavernous hemangiomas of the liver: are there any indications for resection ? World J Surg 19:19-24

20. Di Carlo I, Toro A (2013) Discomfort and anxiety should never be considered surgical indications for hemangioma of the liver. World J Surg 37:2723-2724

21. Di Carlo I, Sofia M, Toro A (2005) Does the psychological request of the patient justify the surgery for hepatic hemangioma? Hepatogastroenterology 52:657-661

22. Vernuccio F, Ronot M, Burgio MD et al (2018) Uncommon evolutions and complications of common benign liver lesions. Abdom Radiol 43:2075-2096

23. Kamyab AA, Rezaei-Kalantari K (2019) Hepatic hemangioma in a cluster of Iranian population. J Med Ultrasound 27:97-100

\section{Publisher's Note}

Springer Nature remains neutral with regard to jurisdictional claims in published maps and institutional affiliations.

\section{Submit your manuscript to a SpringerOpen ${ }^{\circ}$ journal and benefit from:}

- Convenient online submission

- Rigorous peer review

- Open access: articles freely available online

High visibility within the field

- Retaining the copyright to your article

Submit your next manuscript at $>$ springeropen.com 